\title{
Relativistic Alpha Field Theory - Part III: Does Gravitational Force Becomes Positive if $\left(\mathrm{GM} / \mathrm{rc}^{2}\right)>1$ ?
}

\author{
Branko M. Novakovic
}

\begin{abstract}
General Relativity Theory (GRT) cannot be applied to the extremely strong gravitational field at the Planck's scale, because of the related singularity. Here we show that Relativistic Alpha Field (RAF) theory extends the application of GRT to the extremely strong fields at the Planck's scale. This is the consequence of the following predictions of RAF theory: a) no a singularity at the Schwarzschild radius, b) there exists a minimal radius at $r=\left(G M / 2 c^{2}\right)$ that prevents singularity at $r=0$, i.e. the nature protects itself, $c)$ the gravitational force becomes positive (repulsive) if $\left(G M / r c^{2}\right)>1$, that could be a source of a dark energy, and d) unification of electrical and gravitational forces can be done in the standard four dimensions (4D). Predictions a) and b) are presented in the second part of this theory, while predictions c) and d) are considered in this (third) part of the theory. In the first part of the theory we present the solution of the field parameters in RAF theory. In this (third) part of the theory we present the solution of the geodesic equations employing alpha field parameters in RAF theory. This solution for a gravitational field gives the prediction c) of RAF theory, while the related solution for unified electrical and gravitational field gives the prediction d) of RAF theory. If RAF theory is correct, then it could be applied to the both weak and strong fields at the Universe and Planck's scales giving the new light to the regions like black holes, quantum theory, high energy physics, Big Bang theory and cosmology.
\end{abstract}

Index Terms: Relativistic alpha field theory, Field parameters, Geodesic equations, Unification of electrical and gravitational forces

\section{INTRODUCTION}

As it is well known, General Relativity Theory (GRT) [1-6] cannot be applied to the extremely strong gravitational field at the Planck's scale, because of the related singularity. Here we present a new theory that is called Relativistic Alpha Field (RAF) theory. We show that RAF theory extends the capability of the GRT for the application to the extremely strong fields at the Planck's scale. This is the consequence of the following predictions of RAF theory: a) no a singularity at the Schwarzschild radius, b) there exists a minimal radius at $r$ $=\left(G M / 2 c^{2}\right)$ that prevents singularity at $r=0$, i.e. the nature protects itself, c) the gravitational force becomes positive (repulsive) if $\left(G M / r c^{2}\right)>1$, that could be a source of a dark energy in the universe, and d) unification of electrical and gravitational forces can be done in the standard four dimensions (4D). Predictions a) and b) are presented in the second part of this theory, while predictions c) and d) are considered in this (third) part of the theory.

Branko Novakovic, FSB - University of Zagreb, Luciceva 5, P.O.B. 509 10000 Zagreb, Croatia
The solution of the field parameters in RAF theory is presented in the first part of the theory. In this (third) part of the theory we present the solution of the geodesic equations employing alpha field parameters in RAF theory. This solution for a gravitational field gives the prediction c) of RAF theory. The related solution of the geodesic equations for the alpha field parameters of unified electrical and gravitational field gives the prediction d) of RAF theory.

It is also well known, that for unification of the electroweak and strong interactions with gravity, one can use the following two possibilities [1-6]: a) trying to describe gravity as a gauge theory, or b) trying to describe gauge theories as gravity. The first possibility (a) has attracted a lot of attention, but because of the known difficulties, this approach set gravity apart from the standard gauge theories. The second possibility (b) is much more radical. The initial idea has been proposed by Kaluza-Klein theory [7, 8], which today has many variations [9-14], and takes the place in the modern theories like high energy physics (supergravity [15-17] and string theories [18-29]). These theories use five or more extra dimensions with the related dimensional reduction to the four dimensions. Meanwhile, we do not know the answers to the some questions like: can we take the extra dimensions as a real, or as a mathematical device? Here we show that RAF theory predicts the unification of electrical and gravitational forces in the standard four dimensions (4D). This unification is based on the geometric approach. In that sense, the concept of the two dimensionless (unitless) field parameters $\alpha$ and $\alpha^{\prime}$ is introduced in the first part of the theory [30]. These parameters are scalar functions of the potential energy of a particle in electrical, gravitational and unified fields.

RAF theory starts with the main preposition: if the electrical, gravitational and unified fields (forces) can be described by the geometric approach, then the field parameters $\alpha$ and $\alpha^{\prime}$ of a particle in the electrical, gravitational and unified fields should satisfy the Einstein's field equations and the Einstein's geodesic equations. The proposition, related to the satisfaction of the Einstein's field equations is proved in the second part of RAF theory [31]. The proposition, related to the satisfaction of the Einstein's geodesic equations is considered in this (third) part of RAF theory. In the first part of RAF theory [30] we create the field parameters $\alpha$ and $\alpha^{\prime}$ as the dimensionless (unit-less) functions of the potential energy of a particle in an alpha field. This opened the capability of determination of the field parameters $\alpha$ and $\alpha^{\prime}$ of electrical, gravitational and unified electrical and gravitational fields. The obtained relations for the electrical, gravitational and unified forces generally describe the interactions in the strong fields. In the case of the weak fields, the force relations are reduced to the well known 
descriptions. If RAF theory is correct, then it could be applied to the both weak and strong fields at the Universe and Planck's scales giving the new light to the regions like black holes, quantum theory, high energy physics, Big Bang theory and cosmology.

This paper is organized as follows. Sec. II shows the procedure for unification of gravitational and electrical fields. Unification of electrical and gravitational forces in 4D is presented in Sec. III. In the subsection A of Sec. III it is considered the proof of the prediction d) of RAF theory: unification of electrical and gravitational forces can be done in the standard four dimensions (4D). Derivation of electrical force equations is presented in Sec. IV. Derivation of gravitational force equations is pointed out in Sec. V. In the subsection B of Sec. V it is considered the proof of the prediction c) of RAF theory: the gravitational force becomes positive (repulsive) if $\left(G M / r c^{2}\right)>1$. Finally, the related conclusion and the reference list are presented in Sec. VI and Sec. VII, respectively.

\section{UNIFICATION OF GRAVITATIONAL AND ELECTRICAL FIELDS}

The basic problem of this (third) part of RAF theory is to unify the gravitational and electrical forces in the Einstein's four dimension (4D) by using of the gravity (geometric) concept. In order to do this we have to unify gravitational and electrical fields, a priory. In that sense the following propositions are introduced.

(a) Proposition 1. Let $m_{0}$ is a rest mass of a particle, $U$ is a potential energy of the particle in an alpha field, $c$ is the speed of the light in a vacuum and $(i)$ is an imaginary unit. In that case the field parameters $\alpha$ and $\alpha^{\prime}$ can be described as scalar dimensionless (unitless) functions of the potential energy $U$ of a particle in an alpha field [30]. There are four solution for both parameters $\alpha$ and $\alpha^{\prime}$ in an alpha field that can be presented by the following relations:

$$
\begin{aligned}
& f(U)=2 U / m_{0} c^{2}+\left(U / m_{0} c^{2}\right)^{2}, \quad \rightarrow \\
& \alpha_{1,2}=1 \pm i \sqrt{f(U)}, \alpha_{1,2}^{\prime}=1 \mp i \sqrt{f(U)}, \\
& \alpha_{3,4}=-1 \pm i \sqrt{f(U)}, \alpha_{3,4}^{\prime}=-1 \mp i \sqrt{f(U)} .
\end{aligned}
$$

The product of the field parameters $\alpha \alpha^{\prime}$ is an invariant related to the four solutions of the field parameters $\alpha$ and $\alpha^{\prime}$

$$
\alpha_{i} \alpha_{i}^{\prime}=\left(1+\frac{U}{m_{0} c^{2}}\right)^{2}=\alpha \alpha^{\prime}, \quad i=1,2,3,4 .
$$

For calculation some of the quantities in an alpha field we often need to know the difference of the field parameters $\left(\alpha-\alpha^{\prime}\right)$ :

$$
\begin{aligned}
& \alpha_{1}-\alpha_{1}^{\prime}=2 i \sqrt{f(U)}, \quad \alpha_{2}-\alpha_{2}^{\prime}=-2 i \sqrt{f(U)}, \\
& \alpha_{3}-\alpha_{3}^{\prime}=2 i \sqrt{f(U)}, \quad \alpha_{4}-\alpha_{4}^{\prime}=-2 i \sqrt{f(U)}, \rightarrow \\
& \left(\alpha_{1}-\alpha_{1}^{\prime}\right)=\left(\alpha_{3}-\alpha_{3}^{\prime}\right), \quad\left(\alpha_{2}-\alpha_{2}^{\prime}\right)=\left(\alpha_{4}-\alpha_{4}^{\prime}\right) .
\end{aligned}
$$

It should be pointed out that equations (1), (2) and (3) are valid generally and for their calculation we only need to know potential energy $U$ of a particle in the related potential field.
The proof of the proposition 1 has been presented in the first part of this theory [30].

In order to proof that field parameters $\alpha$ and $\alpha^{\prime}$ satisfy of the Einstein's geodesic equations, we can start with the general line element in an alpha field. This line element is described by the nondiagonal form with the Riemannian metrics [32, 33-35]

$$
\begin{gathered}
d s^{2}=-\alpha \alpha^{\prime} c^{2} d t^{2}-\kappa\left(\alpha-\alpha^{\prime}\right)_{x} c d t d x^{1} \\
-\kappa\left(\alpha-\alpha^{\prime}\right)_{y} c d t d x^{2}-\kappa\left(\alpha-\alpha^{\prime}\right)_{z} c d t d x^{3} \\
+\left(d x^{1}\right)^{2}+\left(d x^{2}\right)^{2}+\left(d x^{3}\right)^{2}
\end{gathered}
$$

Here parameter $\kappa$ is a constant with values $\kappa= \pm 1$ (see the first part of this theory [30]). The differential form of the contravariant displacement four-vector in an alpha field, $d X$, can be defined in the frame $\mathrm{K}$ by the relations: $d X \rightarrow K(c d t, d x, d y, d z)=\left\{d x^{\mu}\right\}, \quad \mu=0,1,2,3$,

$d x^{0}=c d t, \quad d x^{1}=d x, \quad d x^{2}=d y, \quad d x^{3}=d z$.

Following the equations (4) and (5) one can derive a matrix expression of the components of the general covariant metric tensor $g_{\mu v}$ valid for the line element (4) and the coordinate system (5)

$\left[g_{\mu v}\right]=\left[\begin{array}{cccc}-v & \lambda_{x} & \lambda_{y} & \lambda_{z} \\ \lambda_{x} & 1 & 0 & 0 \\ \lambda_{y} & 0 & 1 & 0 \\ \lambda_{z} & 0 & 0 & 1\end{array}\right]$

The non-null components of the metric tensor (6) are determined by the following relations:

$$
\begin{aligned}
& g_{00}=-v=-\alpha \alpha^{\prime}, g_{01}=g_{10}=\lambda_{x}=\frac{-\kappa\left(\alpha-\alpha^{\prime}\right)_{x}}{2}, \\
& g_{02}=g_{20}=\lambda_{y}=\frac{-\kappa\left(\alpha-\alpha^{\prime}\right)_{y}}{2}, \\
& g_{03}=g_{30}=\lambda_{z}=\frac{-\kappa\left(\alpha-\alpha^{\prime}\right)_{z}}{2}, g_{11}=1, g_{22}=1, g_{33}=1 .
\end{aligned}
$$

The metric tensor (6) is symmetric and has ten non-zero elements, as we expected that should be. The matrix expression of the metric tensor (6) has nondiagonal form and belongs to the well known Riemannian metrics. Therefore, the related line element (4) is called a nondiagonal line element. The components of the contravariant general metric tensor $g^{\mu v}$ in an alpha field can be derived by inversion of the covariant one using of the matrix (6)

$\left[g^{\mu \nu}\right]=\left[\begin{array}{llll}g^{00} & g^{01} & g^{02} & g^{03} \\ g^{10} & g^{11} & g^{12} & g^{13} \\ g^{20} & g^{21} & g^{22} & g^{23} \\ g^{30} & g^{31} & g^{32} & g^{33}\end{array}\right]$.


Here the values of the elements of the contravariant metric tensor (8) are given as follows:

$$
\begin{aligned}
& g^{00}=\frac{-1}{v+\lambda^{2}}, g^{01}=g^{10}=\frac{\lambda x}{v+\lambda^{2}}, g^{02}=g^{20}=\frac{\lambda y}{v+\lambda^{2}}, \\
& g^{03}=g^{30}=\frac{\lambda z}{v+\lambda^{2}}, g^{11}=\frac{v+\lambda y^{2}+\lambda z^{2}}{v+\lambda^{2}}, g^{12}=g^{21}=\frac{-\lambda x \lambda y}{v+\lambda^{2}}, \\
& g^{13}=g^{31}=\frac{-\lambda x \lambda z}{v+\lambda^{2}}, g^{22}=\frac{v+\lambda x^{2}+\lambda z^{2}}{v+\lambda^{2}}, g^{23}=g^{32}=\frac{-\lambda y \lambda z}{v+\lambda^{2}} \\
& g^{33}=\frac{v+\lambda x^{2}+\lambda y^{2}}{v+\lambda^{2}}, \quad \lambda^{2}=\lambda x^{2}+\lambda y^{2}+\lambda z^{2} .
\end{aligned}
$$

The determinants of the matrices of the metric tensors (6) and (8) are presented by the relations:

$$
\operatorname{det}\left[g_{\mu \nu}\right]=-\left(v+\lambda^{2}\right), \operatorname{det}\left[g^{\mu \nu}\right]=-1 /\left(v+\lambda^{2}\right) \text {. }
$$

Now, we recall the well known condition that should be satisfied by any metric tensor [1-6, 34]

$$
\begin{aligned}
& \sqrt{-\operatorname{det}\left[g_{\mu \nu}\right]}=\sqrt{\nu+\lambda^{2}}=\sqrt{\alpha \alpha^{\prime}+\frac{\kappa^{2}\left(\alpha-\alpha^{\prime}\right)^{2}}{4}}=1, \\
& v+\lambda^{2}=1, \kappa^{2}=1, \rightarrow\left(\frac{\alpha+\alpha^{\prime}}{2}\right)^{2}=1 .
\end{aligned}
$$

The condition (11) gives the important relation between field parameters $\alpha$ and $\alpha^{\prime}$. This relation has been employed in the procedure of determination of the field parameters $\alpha$ and $\alpha^{\prime}$ in the first part of this theory [30]. Including the condition (11) the values of the elements $g^{\mu \nu}$ of the contravariant metric tensor (9) can be presented in the simplified form:

$$
\begin{aligned}
& g^{00}=-1, g^{01}=g^{10}=\lambda x, g^{02}=g^{20}=\lambda y, \\
& g^{03}=g^{30}=\lambda z, \lambda^{2}=\lambda x^{2}+\lambda y^{2}+\lambda z^{2}, \\
& g^{11}=v+\lambda y^{2}+\lambda z^{2}, g^{12}=g^{21}=-\lambda x \lambda y, g^{13}=g^{31}=-\lambda x \lambda z, \\
& g^{22}=v+\lambda x^{2}+\lambda z^{2}, g^{23}=g^{32}=-\lambda y \lambda z, g^{33}=v+\lambda x^{2}+\lambda y^{2} .
\end{aligned}
$$

Further, the simplified form (12) of the elements $g^{\mu v}$ of the contravariant metric tensor (9) has been employed in the following sections of this (third) part of RAF theory.

(b) Proposition 2. If an alpha field is described by the line element (4), then the non zero second type of the Christoffel symbols $\Gamma_{\eta \mu}^{\gamma}$ of the metric tensors (6) and (8) for $\gamma=0$ are given by the following relations:

$$
\begin{aligned}
& \gamma=0 \rightarrow \Gamma_{00}^{0}=-\left(\lambda x^{2} \lambda^{\prime} x+\lambda y^{2} \lambda_{y}^{\prime}+\lambda z^{2} \lambda_{z}^{\prime}\right), \\
& \Gamma_{01}^{0}=\Gamma_{10}^{0}=-\lambda x \lambda^{\prime} x, \quad \Gamma_{02}^{0}=\Gamma_{20}^{0}=-\lambda y \lambda_{y}^{\prime}, \\
& \Gamma_{03}^{0}=\Gamma_{30}^{0}=-\lambda z \lambda_{z}^{\prime}, \Gamma_{11}^{0}=-\lambda_{x}^{\prime}, \Gamma_{22}^{0}=-\lambda^{\prime} y, \\
& \Gamma_{33}^{0}=-\lambda_{z}^{\prime}, \frac{\partial \lambda x}{\partial x}=\lambda_{x}^{\prime}, \frac{\partial \lambda y}{\partial y}=\lambda_{y}^{\prime}, \frac{\partial \lambda z}{\partial z}=\lambda_{z}^{\prime} .
\end{aligned}
$$

The non zero second type of the Christoffel symbols $\Gamma_{\eta \mu}^{\gamma}$ of the metric tensors (6) and (8) for $\gamma=1$ are presented by the expressions:

$$
\begin{aligned}
& \gamma=1 \rightarrow \Gamma_{00}^{1}=\frac{\dot{\lambda} x}{c}-\lambda x \lambda^{\prime}{ }_{x}+\lambda x\left(\lambda x^{2} \lambda_{x}^{\prime}\right. \\
& \left.+\lambda y^{2} \lambda^{\prime} y+\lambda z^{2} \lambda_{z}^{\prime}\right), \quad \Gamma_{01}^{1}=\Gamma_{10}^{1}=\lambda x^{2} \lambda_{x}^{\prime}, \\
& \Gamma_{02}^{1}=\Gamma_{20}^{1}=\lambda x \lambda_{y} \lambda^{\prime} y, \Gamma_{03}^{1}=\Gamma_{30}^{1}=\lambda x \lambda_{z} \lambda_{z}^{\prime}, \\
& \Gamma_{11}^{1}=\lambda x \lambda_{x}^{\prime}, \quad \Gamma_{22}^{1}=\lambda x \lambda_{y,}^{\prime} \Gamma_{33}^{1}=\lambda x \lambda_{z}^{\prime}, \\
& \frac{\partial \lambda x}{\partial c t}=\frac{\dot{\lambda} x}{c}, \quad \frac{\partial \lambda x}{\partial x}=\lambda_{x}^{\prime}, \quad \frac{\partial \lambda y}{\partial y}=\lambda_{y}^{\prime}, \quad \frac{\partial \lambda z}{\partial z}=\lambda_{z}^{\prime} .
\end{aligned}
$$

The non zero second type of the Christoffel symbols $\Gamma_{\eta \mu}^{\gamma}$ of the metric tensors (6) and (8) for $\gamma=2$ are given by the equations:

$\gamma=2 \rightarrow \Gamma_{00}^{2}=\frac{\dot{\lambda} y}{c}-\lambda y \lambda^{\prime} y+\lambda y\left(\lambda x^{2} \lambda^{\prime} x+\lambda y^{2} \lambda^{\prime} y+\lambda z^{2} \lambda_{z}^{\prime}\right)$,

$\Gamma_{01}^{2}=\Gamma_{10}^{2}=\lambda y \lambda x \lambda^{\prime} x, \quad \Gamma_{02}^{2}=\Gamma_{20}^{2}=\lambda y^{2} \lambda^{\prime} y$,

$\Gamma_{03}^{2}=\Gamma_{30}^{2}=\lambda_{y} \lambda_{z} \lambda^{\prime}{ }_{z}, \Gamma_{11}^{2}=\lambda_{y} \lambda^{\prime} x, \Gamma_{22}^{2}=\lambda y \lambda^{\prime} y, \Gamma_{33}^{2}=\lambda y \lambda^{\prime} z$,

$\frac{\partial \lambda_{y}}{\partial c t}=\frac{\dot{\lambda} y}{c}, \quad \frac{\partial \lambda_{x}}{\partial x}=\lambda_{x}^{\prime}, \quad \frac{\partial \lambda_{y}}{\partial y}=\lambda_{y}^{\prime}, \quad \frac{\partial \lambda_{z}}{\partial z}=\lambda_{z}^{\prime}$.

Finally, the non zero second type of the Christoffel symbols $\Gamma_{\eta \mu}^{\gamma}$ of the metric tensors (6) and (8) for $\gamma=3$ are presented by the following relations:

$\gamma=3 \rightarrow \Gamma_{00}^{3}=\frac{\dot{\lambda} z}{c}-\lambda_{z} \lambda_{z}^{\prime}+\lambda_{z}\left(\lambda_{x}{ }^{2} \lambda^{\prime}{ }_{x}+\lambda_{y}{ }^{2} \lambda^{\prime} y+\lambda_{z}{ }^{2} \lambda_{z}^{\prime}\right)$,

$\Gamma_{01}^{3}=\Gamma_{10}^{3}=\lambda_{z} \lambda_{x} \lambda^{\prime}{ }_{x}, \Gamma_{02}^{3}=\Gamma_{20}^{3}=\lambda_{z} \lambda_{y} \lambda^{\prime}{ }_{y}$,

$\Gamma_{03}^{3}=\Gamma_{30}^{3}=\lambda_{z}{ }^{2} \lambda^{\prime}{ }_{z}, \Gamma_{11}^{3}=\lambda_{z} \lambda^{\prime} x, \Gamma_{22}^{3}=\lambda_{z} \lambda^{\prime} y, \Gamma_{33}^{3}=\lambda_{z} \lambda_{z}^{\prime}$,

$\frac{\partial \lambda_{z}}{\partial c t}=\frac{\dot{\lambda}_{z}}{c}, \quad \frac{\partial \lambda_{x}}{\partial x}=\lambda_{x}^{\prime}, \quad \frac{\partial \lambda_{y}}{\partial y}=\lambda_{y}^{\prime}, \quad \frac{\partial \lambda_{z}}{\partial z}=\lambda_{z}^{\prime}$.

(c) Proof of the proposition 2. In order to prove of the proposition 2, we can start with the well-known relation for calculation of the second type Christoffel symbols [1-6] of the metric tensors (6) and (8)

$$
\Gamma_{\eta \mu}^{\gamma}=\frac{g^{\kappa \gamma}}{2}\left[g_{\kappa \eta, \mu}+g_{\kappa \mu, \eta}-g_{\eta \mu, \kappa}\right], \kappa, \gamma, \eta, \mu=0,1,2,3 \text {. }
$$

Thus, if an alpha field is described by the line element (4), then the non zero second type of the Christoffel symbols $\Gamma_{\eta \mu}^{\gamma}$ of the metric tensors (6) and (8) for $\gamma=0$ are given by the following relations: 
$\gamma=0 \rightarrow \Gamma_{00}^{0}=\frac{1}{2}\left[\begin{array}{l}\frac{\dot{v}}{c}+\lambda_{x}\left(2 \frac{\dot{\lambda} x}{c}+v^{\prime} x\right)+\lambda_{y}\left(2 \frac{\dot{\lambda} y}{c}+v^{\prime} y\right) \\ +\lambda_{z}\left(2 \frac{\dot{\lambda} z}{c}+v_{z}^{\prime}\right)\end{array}\right]$,

$\Gamma_{01}^{0}=\Gamma_{10}^{0}=\frac{v^{\prime} x}{2}, \Gamma_{02}^{0}=\Gamma_{20}^{0}=\frac{v^{\prime} y}{2}, \Gamma_{03}^{0}=\Gamma_{30}^{0}=\frac{v^{\prime} z}{2}, \Gamma_{11}^{0}=-\lambda^{\prime} x$

$\Gamma_{22}^{0}=-\lambda_{y}^{\prime}, \Gamma_{33}^{0}=-\lambda_{z}^{\prime}, \frac{\partial v}{\partial c t}=\frac{\dot{v}}{c}, \frac{\partial \lambda_{x}}{\partial c t}=\frac{\dot{\lambda}_{x}}{c}, \frac{\partial \lambda_{y}}{\partial c t}=\frac{\dot{\lambda}_{y}}{c}, \frac{\partial \lambda_{z}}{\partial c t}=\frac{\dot{\lambda}_{z}}{c}$,

$\frac{\partial v}{\partial x}=v^{\prime} x, \frac{\partial v}{\partial y}=v_{y}^{\prime}, \frac{\partial v}{\partial z}=v_{z}^{\prime}, \frac{\partial \lambda_{x}}{\partial x}=\lambda_{x}^{\prime} x, \frac{\partial \lambda_{y}}{\partial y}=\lambda_{y}^{\prime}, \frac{\partial \lambda_{z}}{\partial z}=\lambda_{z}^{\prime}$.

The non zero second type of the Christoffel symbols $\Gamma_{\eta \mu}^{\gamma}$ of the metric tensors (6) and (8) for $\gamma=1$ are given by the equations:

$\gamma=1 \rightarrow \Gamma_{00}^{1}=\frac{1}{2}\left[\begin{array}{c}-\frac{\dot{v}}{c} \lambda x+\left(v+\lambda y^{2}+\lambda z^{2}\right)\left(2 \frac{\dot{\lambda} x}{c}+v^{\prime} x\right)- \\ -\lambda x \lambda y\left(2 \frac{\dot{\lambda} y}{c}+v^{\prime} y\right)-\lambda x \lambda z\left(2 \frac{\dot{\lambda} z}{c}+v^{\prime} z\right)\end{array}\right]$,
$\Gamma_{01}^{1}=\Gamma_{10}^{1}=\frac{-\lambda x v^{\prime} x}{2}, \Gamma_{02}^{1}=\Gamma_{20}^{1}=\frac{-\lambda x v^{\prime} y}{2}$,
$\Gamma_{03}^{1}=\Gamma_{30}^{1}=\frac{-\lambda x v^{\prime} z}{2}, \Gamma_{11}^{1}=\lambda x \lambda_{x}^{\prime} x, \Gamma_{22}^{1}=\lambda x \lambda_{y}^{\prime}, \Gamma_{33}^{1}=\lambda x \lambda_{z}^{\prime}$.

The non zero second type of the Christoffel symbols $\Gamma_{\eta \mu}^{\gamma}$ of the metric tensors (6) and (8) for $\gamma=2$ are given by the relations:

$$
\gamma=2 \rightarrow \Gamma_{00}^{2}=\frac{1}{2}\left[\begin{array}{l}
-\frac{\dot{v}}{c} \lambda y-\lambda x \lambda y\left(2 \frac{\dot{\lambda} x}{c}+v^{\prime} x\right) \\
\left.+v+\lambda x^{2}+\lambda z^{2}\right)\left(2 \frac{\dot{\lambda} y}{c}+v^{\prime} y\right) \\
\Gamma_{11}^{2}=\lambda \lambda^{\prime} \lambda_{x}^{\prime}, \Gamma_{22}^{2}=\lambda_{y} \lambda^{\prime} y, \Gamma_{33}^{2}=\lambda \lambda_{y} \lambda_{z}^{\prime}, \Gamma_{01}^{2}=\Gamma_{10}^{2}=\frac{-\lambda y v^{\prime} x}{2}, \\
\Gamma_{02}^{2}=\Gamma_{20}^{2}=\frac{-\lambda y v^{\prime} y}{2}, \Gamma_{03}^{2}=\Gamma_{30}^{2}=\frac{-\lambda y v^{\prime} z}{2} .
\end{array}\right]
$$

Finally, the non zero second type of the Christoffel symbols $\Gamma_{\eta \mu}^{\gamma}$ of the metric tensors (6) and (8) for $\gamma=3$ are given by the following expressions:

$$
\begin{aligned}
& \gamma=3 \rightarrow \Gamma_{00}^{3}=\frac{1}{2}\left[\begin{array}{c}
-\frac{\dot{v}}{c} \lambda_{z}-\lambda x \lambda z\left(2 \frac{\dot{\lambda} x}{c}+v^{\prime} x\right)-\lambda y \lambda z\left(2 \frac{\dot{\lambda} y}{c}+v^{\prime} y\right) \\
+\left(v+\lambda x^{2}+\lambda_{y}^{2}\right)\left(2 \frac{\dot{\lambda} z}{c}+v_{z}^{\prime}\right)
\end{array}\right], \\
& \Gamma_{11}^{3}=\lambda_{z} \lambda_{x}^{\prime}, \Gamma_{22}^{3}=\lambda_{z} \lambda_{y}^{\prime}, \Gamma_{33}^{3}=\lambda_{z} \lambda_{z}^{\prime}, \Gamma_{01}^{3}=\Gamma_{10}^{3}=\frac{-\lambda z v^{\prime} x}{2}, \\
& \Gamma_{02}^{3}=\Gamma_{20}^{3}=\frac{-\lambda z v^{\prime} y}{2}, \quad \Gamma_{03}^{3}=\Gamma_{30}^{3}=\frac{-\lambda_{z} v_{z}^{\prime}}{2} .
\end{aligned}
$$

Now, including the condition (11) we can derive the following relations:

$$
\begin{aligned}
& v+\lambda^{2}=1 \rightarrow v=1-\lambda^{2}=1-\left(\lambda x^{2}+\lambda y^{2}+\lambda z^{2}\right), \\
& \frac{\dot{v}}{c}=-2\left(\lambda x \frac{\dot{\lambda} x}{c}+\lambda y \frac{\dot{\lambda}_{y}}{c}+\lambda z \frac{\dot{\lambda} z}{c}\right), \\
& v^{\prime}=-2\left(\lambda x \lambda^{\prime} x+\lambda y \lambda^{\prime} y+\lambda z \lambda^{\prime} z\right) .
\end{aligned}
$$

If we apply the relations given by (22), then the Christoffel symbols from the previous equations become the functions of the parameter $\lambda$ and its time and space partial derivations, $\dot{\lambda}$ and $\lambda^{\prime}$, only. In that case the non zero second type of the Christoffel symbols of the metric tensors (6) and (8) for $\gamma=0,1,2,3$ shown by the equations (18), (19), (20) and (21) are transformed into the simplest relations given by (13), (14), (15) and (16), respectively. On that way the proof of the proposition 2 is finished.

(d) Proposition 3. If an alpha field is described by the line element (4), then the geodesic equations, related to the non zero second type of the Christoffel symbols $\Gamma_{\eta \mu}^{\gamma}$ from the equations (13) to (16), have the forms given by the relations (23) and (24). Thus, the geodesic equation for the coordinate $\mathrm{x}^{0}=\mathrm{ct}$ is described by the relation:

$$
\begin{aligned}
& x^{0}=c t, \quad \dot{x}^{0}=c, \quad \ddot{x}^{0}=0, \rightarrow \\
& A=\lambda^{\prime} x(\lambda x c+\dot{x})^{2}+\lambda^{\prime} y\left(\lambda_{y} c+\dot{y}\right)^{2}+\lambda_{z}^{\prime}\left(\lambda_{z} c+\dot{z}\right)^{2}=0 .
\end{aligned}
$$

On the other hand, the geodesic equations for the coordinates $x^{1}=x, x^{2}=y, x^{3}=z$ are described by the relations:

$$
\begin{aligned}
& \ddot{x}+\dot{\lambda}_{x} c-\lambda_{x} \lambda^{\prime}{ }_{x} c^{2}=0, \quad \ddot{y}+\dot{\lambda}_{y} c-\lambda_{y} \lambda^{\prime} y c^{2}=0, \\
& \ddot{z}+\dot{\lambda}_{z} c-\lambda_{z} \lambda^{\prime}{ }_{z} c^{2}=0 .
\end{aligned}
$$

For the time-invariant, or very slowly changeable alpha field, the parameters $\dot{\lambda}_{x}=\dot{\lambda}_{y}=\dot{\lambda}_{z}=0$, and the relations 24 are transformed into the simplest forms:

$$
\ddot{x}-\lambda x \lambda_{x}^{\prime} c^{2}=0, \ddot{y}-\lambda_{y} \lambda^{\prime}{ }_{y} c^{2}=0, \ddot{z}-\lambda_{z} \lambda^{\prime}{ }_{z} c^{2}=0 \text {. }
$$

The related force equations for a particle with rest mass $m_{0}$ can be obtained by using of the relations (24):

$$
\begin{aligned}
& F_{x}=-m_{0} \dot{\lambda}_{x} c+m_{0} \lambda_{x} \lambda^{\prime}{ }_{x} c^{2}, F_{y}=-m_{0} \dot{\lambda}_{y} c+m_{0} \lambda_{y} \lambda^{\prime} c^{2}, \\
& F_{z}=-m_{0} \dot{\lambda}_{z} c+m_{0} \lambda_{z} \lambda^{\prime}{ }_{z} c^{2} .
\end{aligned}
$$


For the time-invariant, or very slowly changeable alpha field, the force relations (26) are transformed into the simplest forms:

$$
F_{x}=m_{0} \lambda_{x} \lambda^{\prime}{ }_{x} c^{2}, \quad F_{y}=m_{0} \lambda_{y} \lambda^{\prime} y c^{2}, \quad F_{z}=m_{0} \lambda_{z} \lambda_{z}^{\prime} c^{2} .
$$

(e) Proof of the proposition 3. In order to prove of the proposition 3, we can start with the well-known relation for calculation of the geodesic equations [1-6]:

$$
\ddot{x}^{\gamma}+\Gamma_{\eta \mu}^{\gamma} \dot{x}^{\eta} \dot{x}^{\mu}=0, \quad \gamma, \eta, \mu=0,1,2,3 \text {. }
$$

Applying (28) and parameter $\gamma=0$ we obtain the first geodesic equation

$$
\begin{gathered}
\gamma=0 \rightarrow \ddot{x}^{0}-\left(\lambda x^{2} \lambda^{\prime}{ }_{x}+\lambda y^{2} \lambda^{\prime}{ }_{y}+\lambda z^{2} \lambda_{z}^{\prime}\right)\left(\dot{x}^{0}\right)^{2} \\
-2\left(\lambda x \lambda^{\prime}{ }_{x}\right) \dot{x}^{0} \dot{x}^{1}-2\left(\lambda_{y} \lambda^{\prime} y\right) \dot{x}^{0} \dot{x}^{2}-2\left(\lambda_{z} \lambda_{z}^{\prime}\right) \dot{x}^{0} \dot{x}^{3} \\
-\lambda_{x}^{\prime}\left(\dot{x}^{1}\right)^{2}-\lambda^{\prime}{ }_{y}\left(\dot{x}^{2}\right)^{2}-\lambda_{z}^{\prime}\left(\dot{x}^{3}\right)^{2}=0 .
\end{gathered}
$$

Taking into the account parameter $\gamma=1$ and the relation (28) we obtain the second geodesic equation

$$
\begin{aligned}
& \gamma=1, \rightarrow \ddot{x}^{1}+\left[\begin{array}{l}
\frac{\dot{\lambda} x}{c}-\lambda x \lambda^{\prime} x \\
+\lambda x\left(\lambda x^{2} \lambda^{\prime}{ }_{x}+\lambda y^{2} \lambda^{\prime} y+\lambda z^{2} \lambda^{\prime} z\right)
\end{array}\right]\left(\dot{x}^{0}\right)^{2} \\
& +2\left(\lambda x^{2} \lambda^{\prime}{ }_{x}\right) \dot{x}^{0} \dot{x}^{1}+2\left(\lambda x \lambda_{y} \lambda^{\prime}{ }_{y}\right) \dot{x}^{0} \dot{x}^{2}+2\left(\lambda x \lambda_{z} \lambda^{\prime}{ }_{z}\right) \dot{x}^{0} \dot{x}^{3} \\
& +\left(\lambda_{x} \lambda^{\prime}{ }_{x}\right)\left(\dot{x}^{1}\right)^{2}+\left(\lambda x \lambda^{\prime}{ }_{y}\right)\left(\dot{x}^{2}\right)^{2}+\left(\lambda x \lambda^{\prime} z\right)\left(\dot{x}^{3}\right)^{2}=0 .
\end{aligned}
$$

If we include parameter $\gamma=2$ into the relation (28), then we obtain the third geodesic equation

$$
\begin{aligned}
& \gamma=2 \rightarrow \ddot{x}^{2}+\left[\begin{array}{l}
\frac{\dot{\lambda} y}{c}-\lambda y \lambda_{y}^{\prime} \\
+\lambda y\left(\lambda x^{2} \lambda^{\prime}{ }_{x}+\lambda y^{2} \lambda_{y}^{\prime}+\lambda z^{2} \lambda_{z}^{\prime}\right)
\end{array}\right]\left(\dot{x}^{0}\right)^{2} \\
& +2\left(\lambda_{y} \lambda_{x} \lambda^{\prime}{ }_{x}\right) \dot{x}^{0} \dot{x}^{1}+2\left(\lambda_{y}{ }^{2} \lambda^{\prime}{ }_{y}\right) \dot{x}^{0} \dot{x}^{2}+2\left(\lambda_{y} \lambda_{z} \lambda^{\prime}{ }_{z}\right) \dot{x}^{0} \dot{x}^{3} \\
& +\left(\lambda_{y} \lambda^{\prime}{ }_{x}\right)\left(\dot{x}^{1}\right)^{2}+\left(\lambda_{y} \lambda_{y}^{\prime}\right)\left(\dot{x}^{2}\right)^{2}+\left(\lambda_{y} \lambda_{z}^{\prime}\right)\left(\dot{x}^{3}\right)^{2}=0 .
\end{aligned}
$$

Finally, if we include parameter $\gamma=3$ into the relation (28), then we obtain the fourth geodesic equation

$$
\begin{aligned}
& \gamma=3 \rightarrow \ddot{x}^{3}+\left[\begin{array}{l}
\frac{\dot{\lambda} z}{c}-\lambda_{z} \lambda^{\prime}{ }_{z} \\
+\lambda_{z}\left(\lambda x^{2} \lambda^{\prime}{ }_{x}+\lambda_{y}{ }^{2} \lambda^{\prime}{ }_{y}+\lambda_{z}{ }^{2} \lambda^{\prime}{ }_{z}\right)
\end{array}\right]\left(\dot{x}^{0}\right)^{2} \\
& +2\left(\lambda_{z} \lambda_{x} \lambda^{\prime}{ }_{x}\right) \dot{x}^{0} \dot{x}^{1}+2\left(\lambda_{z} \lambda_{y} \lambda^{\prime}{ }_{y}\right) \dot{x}^{0} \dot{x}^{2}+2\left(\lambda_{z^{2}}{ }^{\prime} \lambda_{z}\right) \dot{x}^{0} \dot{x}^{3} \\
& +\left(\lambda_{z} \lambda^{\prime}{ }_{x}\right)\left(\dot{x}^{1}\right)^{2}+\left(\lambda_{z} \lambda^{\prime}{ }_{y}\right)\left(\dot{x}^{2}\right)^{2}+\left(\lambda_{z} \lambda^{\prime}{ }_{z}\right)\left(\dot{x}^{3}\right)^{2}=0 .
\end{aligned}
$$

Now, we can introduce the following coordinate system into the previous geodesic equations:

$$
\begin{array}{ll}
\left(x^{0}, x^{1}, x^{2}, x^{3}\right) & \leftrightarrow \quad(c t, x, y, z,), \\
\dot{x}^{0}=c, \quad \dot{x}^{1}=\dot{x}, & \dot{x}^{2}=\dot{y}, \quad \dot{x}^{3}=\dot{z}, \\
\ddot{x}^{0}=0, \quad \ddot{x}^{1}=\ddot{x}, & \ddot{x}^{2}=\ddot{y}, \quad \ddot{x}^{3}=\ddot{z} .
\end{array}
$$

Applying the relations (33) to (29) we obtain the new form of the geodesic equation for the coordinate $x^{0}=c t$ (the first geodesic equation)

$$
\begin{gathered}
\gamma=0 \rightarrow\left(\lambda x^{2} \lambda^{\prime}{ }_{x}+\lambda y^{2} \lambda^{\prime} y+\lambda z^{2} \lambda^{\prime} z\right) c^{2} \\
+2\left(\lambda x \lambda^{\prime}{ }_{x}\right) c \dot{x}+2\left(\lambda_{y} \lambda_{y}^{\prime}\right) c \dot{y}+2\left(\lambda_{z} \lambda_{z}^{\prime}\right) c \dot{z} \\
+\lambda_{x}^{\prime}(\dot{x})^{2}+\lambda^{\prime} y(\dot{y})^{2}+\lambda^{\prime} z(\dot{z})^{2}=0 .
\end{gathered}
$$

This relation can be transformed into the form of the first geodesic equation, given by parameter $A$ in (23). Thus, the geodesic equation for the coordinate $x^{0}=c t$ (the first geodesic equation) given by (23) is proved.

Further, applying (33) to (30) we obtain the new relation of the geodesic equation for the coordinate $x^{1}=x$ (the second geodesic equation)

$$
\gamma=1 \rightarrow \ddot{x}+\left[\begin{array}{l}
\frac{\dot{\lambda} x}{c}-\lambda x \lambda^{\prime} x \\
+\lambda x\left(\lambda x^{2} \lambda^{\prime} x+\lambda y^{2} \lambda^{\prime} y+\lambda z^{2} \lambda^{\prime} z\right)
\end{array}\right] c^{2}
$$

$+2\left(\lambda x^{2} \lambda_{x}^{\prime}\right) c \dot{x}+2\left(\lambda_{x} \lambda_{y} \lambda^{\prime}{ }_{y}\right) c \dot{y}+2\left(\lambda_{x} \lambda_{z} \lambda^{\prime} z\right) c \dot{z}$

$+\left(\lambda x \lambda^{\prime}{ }_{x}\right)(\dot{x})^{2}+\left(\lambda x \lambda^{\prime} y\right)(\dot{y})^{2}+\left(\lambda x \lambda^{\prime} z\right)(\dot{z})^{2}=0$.

Including the condition given by (23) $(A=0)$ this relation can be transformed into the new form of the second geodesic equation, given by the first relation in (24). On that way, the geodesic equation for the coordinate $x^{1}=x$ (the second geodesic equation) is proved.

The new relation of the geodesic equation for the coordinate $x^{2}=y$ (the third geodesic equation) can be obtained by applying (33) to (31)

$$
\begin{aligned}
& \gamma=2 \rightarrow \ddot{y}+\left[\begin{array}{l}
\frac{\dot{\lambda}_{y}}{c}-\lambda \lambda_{y} \lambda_{y} \\
+\lambda_{y}\left(\lambda x^{2} \lambda^{\prime}{ }_{x}+\lambda y^{2} \lambda^{\prime} y+\lambda z^{2} \lambda_{z}^{\prime}\right)
\end{array}\right] c^{2} \\
& +2\left(\lambda_{y} \lambda_{x} \lambda^{\prime}{ }_{x}\right) c \dot{x}+2\left(\lambda_{y}{ }^{2} \lambda^{\prime} y\right) c \dot{y}+2\left(\lambda_{y} \lambda_{z} \lambda^{\prime} z\right) c \dot{z} \\
& +\left(\lambda_{y} \lambda^{\prime}{ }_{x}\right)(\dot{x})^{2}+\left(\lambda y \lambda^{\prime}{ }_{y}\right)(\dot{y})^{2}+\left(\lambda_{y} \lambda^{\prime} z\right)(\dot{z})^{2}=0 .
\end{aligned}
$$

Including the condition given by $(23)(A=0)$ this relation can be transformed into the new form of the third geodesic equation, given by the second relation in (24). Thus, the form of the geodesic equation for the coordinate $x^{2}=y$ (the third geodesic equation) is proved.

Applying (33) to (32) we obtain the new relation of the geodesic equation for the coordinate $x^{3}=z$ (the fourth geodesic equation)

$$
\begin{aligned}
& \gamma=3 \rightarrow \ddot{z}+\left[\begin{array}{l}
\frac{\dot{\lambda} z}{c}-\lambda_{z} \lambda_{z}^{\prime} \\
+\lambda z\left(\lambda x^{2} \lambda^{\prime}{ }_{x}+\lambda_{y}{ }^{2} \lambda^{\prime}{ }_{y}+\lambda_{z}{ }^{2} \lambda^{\prime} z\right)
\end{array}\right] c^{2} \\
& +2\left(\lambda_{z} \lambda_{x} \lambda^{\prime}{ }_{x}\right) c \dot{x}+2\left(\lambda_{z} \lambda_{y} \lambda^{\prime}{ }_{y}\right) c \dot{y}+2\left(\lambda_{z}{ }^{2} \lambda^{\prime} z\right) c \dot{z} \\
& +\left(\lambda_{z} \lambda^{\prime}{ }_{x}\right)(\dot{x})^{2}+\left(\lambda_{z} \lambda^{\prime}{ }_{y}\right)(\dot{y})^{2}+\left(\lambda_{z} \lambda^{\prime}{ }_{z}\right)(\dot{z})^{2}=0 .
\end{aligned}
$$

Including the condition (23) $(A=0)$ this relation can be transformed into the new form of the fourth geodesic equation, given by the third relation in (24). On that way, the 
geodesic equation for the coordinate $x^{3}=z$ (the fourth geodesic equation) is proved.

Finally, multiplying the relations (24) and (25) by the rest mass $m_{0}$ of a particle in an alpha field, we obtain the related force equations given by (26) and (27), respectively. On that way the proof of the proposition 3 is finished.

In order to apply the force relations (26) and (27) to an alpha field we have to know the parameter $\lambda$ and its partial derivations in space $\lambda^{\prime}$ and time $\dot{\lambda}$. Applying (3) and (7) we obtain the following equations for calculation of the parameters $\lambda, \lambda^{\prime}$ and $\dot{\lambda}$ in an alpha field:

$$
\begin{aligned}
& \lambda= \pm i k \sqrt{\frac{2 U}{m_{0} c^{2}}+\left(\frac{U}{m_{0} c^{2}}\right)^{2}}, \sqrt{\cdot}=\sqrt{\frac{2 U}{m_{0} c^{2}}+\left(\frac{U}{m_{0} c^{2}}\right)^{2}}, \\
& \lambda^{\prime}=\frac{\partial \lambda}{\partial r}= \pm \frac{i k}{\sqrt{\cdot}}\left[\frac{1}{m_{0} c^{2}} \frac{\partial U}{\partial r}\left(1+\frac{U}{m_{0} c^{2}}\right)\right], \\
& \dot{\lambda}=\frac{\partial \lambda}{\partial t}= \pm \frac{i k}{\sqrt{\cdot}}\left[\frac{1}{m_{0} c^{2}} \frac{\partial U}{\partial t}\left(1+\frac{U}{m_{0} c^{2}}\right)\right] .
\end{aligned}
$$

Here $U$ and $m_{0}$ are the potential energy and the rest mass of the particle in an alpha field. The related components of the parameters $\lambda, \lambda^{\prime}$ and $\dot{\lambda}$ in $x, y$ and $z$ directions are given by the following equations:

$$
\begin{aligned}
& \lambda x=\lambda \frac{x}{r}, \lambda y=\lambda \frac{y}{r}, \lambda_{z}=\lambda \frac{z}{r}, \lambda^{\prime} x=\lambda^{\prime} \frac{x}{r}, \lambda^{\prime} y=\lambda^{\prime} \frac{y}{r}, \\
& \lambda_{z}^{\prime}=\lambda^{\prime} \frac{z}{r}, \quad \dot{\lambda}_{x}=\dot{\lambda} \frac{x}{r}, \quad \dot{\lambda}_{y}=\dot{\lambda} \frac{y}{r}, \quad \dot{\lambda}_{z}=\dot{\lambda} \frac{z}{r} .
\end{aligned}
$$

\section{UNIFICATION OF ELECTRICAL AND GRAVITATIONAL FORCES}

Let the source of the unified electrical and gravitational fields is an object with mass $M$, electric point charge $Q$ and radius $r$. Thus, if a particle is an electron with a rest mass $m_{0}$ and an electric charge $q$, then the potential energy of the electron in the unified field $U$ is described by the relation [37-39]

$$
U=U_{e}+U_{g}=\frac{q Q}{r}-\frac{m_{0} G M}{r}=q A_{e 0}+m_{0} A_{g 0} .
$$

Here $U_{e}$ is the potential energy of a particle in an electrical field, $U_{g}$ is the potential energy of the particle in a gravitational field, $A_{e 0}$ is a scalar electric potential, $A_{g 0}$ is a scalar gravitational potential and $G$ is a gravitational constant. The potential energy function $f(U)$ for this unified field can be obtained by using equations (1) and (40):

$$
\begin{aligned}
& f(U)=\frac{2 U}{m_{0} c^{2}}+\left(\frac{U}{m_{0} c^{2}}\right)^{2}, \frac{U}{m_{0} c^{2}}=\frac{q Q}{m_{0} r c^{2}}-\frac{m_{0} G M}{m_{0} r c^{2}} \\
& =\frac{G_{e} Q}{r c^{2}}-\frac{G M}{r c^{2}}=\frac{M_{e g}}{r c^{2}}, \quad G_{e}=\frac{q}{m_{0}} \text {, } \\
& M_{e g}=G_{e} Q-G M, \rightarrow f(U)=\frac{2 M_{e g}}{r c^{2}}+\left(\frac{M_{e g}}{r c^{2}}\right)^{2} \text {. }
\end{aligned}
$$

Here parameter $G_{e}=q / m_{0}$ is the Kaluza-Klein constant [6-8], obtained here on the natural way. Now including equations (40) and (41) into (38) we obtain the following relations for the parameters $\lambda, \lambda^{\prime}$ and $\dot{\lambda}$, valid for the unified field:

$$
\begin{aligned}
& \lambda= \pm i k \sqrt{\frac{2 M_{e g}}{r c^{2}}\left(1+\frac{M_{e g}}{2 r c^{2}}\right)}, \lambda^{\prime}=\mp \frac{i k}{\sqrt{\cdot}}\left[\frac{M_{e g}}{r^{2} c^{2}}\left(1+\frac{M_{e g}}{r c^{2}}\right)\right] \\
& \dot{\lambda}= \pm i k \frac{\partial}{\partial t} \sqrt{\frac{2 M_{e g}}{r c^{2}}\left(1+\frac{M_{e g}}{2 r c^{2}}\right)}, \sqrt{\cdot}=\sqrt{\frac{2 M_{e g}}{r c^{2}}\left(1+\frac{M_{e g}}{2 r c^{2}}\right)} .
\end{aligned}
$$

Thus, including (42) and (39) into (24) we obtain the geodesic equations valid for the unified field. Further, including (42) and (39) into (24), with the substitution $M_{e g} \rightarrow M_{e}=G_{e} Q$, we obtain the geodesic equations valid for the electrical field. Finally, including (42) and (39) into (24), with the substitution $M_{e g} \rightarrow M_{g}=-G M$, we obtain the geodesic equations valid for the gravitational field.

Following the relations (42), (39) and (24), and the mentioned substitutions, we can see that the field parameters $\alpha$ and $\alpha^{\prime}$ for the electrical field, gravitational field and unified electrical and gravitational field satisfy the Einstein's geodesic equations. On that way the proof of the satisfaction of the Einstein's geodesic equations, is finished.

Now, applying (42) and (39) to (26) we obtain the force equations for a particle rest mass $m_{0}$ valid for the unified electrical and gravitational field:

$$
\begin{aligned}
\dot{\lambda} \neq 0, & \rightarrow F_{x}=m_{0} \ddot{x}=\mp i k m_{0} \frac{\partial}{\partial t}\left(\sqrt{\frac{2 M_{e g}}{r c^{2}}\left(1+\frac{M_{e g}}{2 r c^{2}}\right)}\right) \frac{x}{r} c \\
& +\frac{m_{0} M_{e g}}{r^{2}}\left(1+\frac{M_{e g}}{r c^{2}}\right) \frac{x}{r}, \\
F_{y}= & m_{0} \ddot{y}=\mp i k m_{0} \frac{\partial}{\partial t}\left(\sqrt{\frac{2 M_{e g}}{r c^{2}}\left(1+\frac{M_{e g}}{2 r c^{2}}\right)}\right) \frac{y}{r} c \\
& +\frac{m_{0} M_{e g}}{r^{2}}\left(1+\frac{M_{e g}}{r c^{2}}\right) \frac{y}{r}, \\
F_{z}= & m_{0} \ddot{z}=\mp i k m_{0} \frac{\partial}{\partial t}\left(\sqrt{\frac{2 M_{e g}}{r c^{2}}\left(1+\frac{M_{e g}}{2 r c^{2}}\right)}\right) \frac{z}{r} c \\
& +\frac{m_{0} M_{e g}}{r^{2}}\left(1+\frac{M_{e g}}{r c^{2}}\right) \frac{z}{r} .
\end{aligned}
$$

For the time-invariant (or very slowly changed) alpha field the parameters $\dot{\lambda}_{x}=\dot{\lambda}_{y}=\dot{\lambda}_{z}=0$. In that case we should apply the relations (27) for derivation of the force equations for the unified electrical and gravitational field: 


$$
\begin{aligned}
& \dot{\lambda}=0, \rightarrow F_{x}=m_{0} \ddot{x}=\frac{m_{0} M_{e g}}{r^{2}}\left(1+\frac{M_{e g}}{r c^{2}}\right) \frac{x}{r}, \\
& F_{y}=m_{0} \ddot{y}=\frac{m_{0} M_{e g}}{r^{2}}\left(1+\frac{M_{e g}}{r c^{2}}\right) \frac{y}{r}, \\
& F_{z}=m_{0} \ddot{z}=\frac{m_{0} M_{e g}}{r^{2}}\left(1+\frac{M_{e g}}{r c^{2}}\right) \frac{z}{r} .
\end{aligned}
$$

Thus, the equations (43) and (44) show that the gravitational and electrical forces are unified in the Einstein's for dimension (4D) by using of the gravity (geometric) concept. An analog approach to derivation of the unified electromagnetic and gravitational forces in 4D has been realized by using generalized relativistic Hamiltonian in an alpha field $[36,46]$.

\section{A. Proof of the Prediction d) of RAF Ttheory}

In prediction d) RAF theory predicted: unification of electrical and gravitational forces can be done in the standard four dimensions (4D). Following the previous consideration we can conclude that the gravitational and electrical forces can be unified in the standard for dimension (4D) by using of the gravity (geometric) concept. The main consequence of this approach is that the obtained relations for the unified forces generally describe the interactions in the strong fields. In the case of the weak fields the force relations are reduced to the well known descriptions of the interactions in the weak fields.

The previous consideration theoretically confirms prediction d) of RAF theory: unification of electrical and gravitational forces can be done in the standard four dimensions (4D). Of course, this should be proved by the related experiments.

\section{DERIVATION Of ElECtricAl ForCE EQUATIONS}

Applying the substitution $M_{e g} \rightarrow M_{e}=G_{e} Q$ to the relations (43), we obtain the force equations for a particle rest mass $m_{0}$ valid for the electrical field:

$$
\begin{aligned}
& \dot{\lambda} \neq 0, F_{x}=m_{0} \ddot{x}=\mp i k m_{0} \frac{\partial}{\partial t}\left(\sqrt{\frac{2 G_{e} Q}{r c^{2}}\left(1+\frac{G_{e} Q}{2 r c^{2}}\right)}\right) \frac{x}{r} c \\
& +\frac{m_{0} G_{e} Q}{r^{2}}\left(1+\frac{G_{e} Q}{r c^{2}}\right) \frac{x}{r}, \\
& F_{y}=m_{0} \ddot{y}=\mp i k m_{0} \frac{\partial}{\partial t}\left(\sqrt{\frac{2 G_{e} Q}{r c^{2}}\left(1+\frac{G_{e} Q}{2 r c^{2}}\right)}\right) \frac{y}{r} c \\
& +\frac{m_{0} G_{e} Q}{r^{2}}\left(1+\frac{G_{e} Q}{r c^{2}}\right) \frac{y}{r}, \\
& F_{z}=m_{0} \ddot{z}=\mp i k m_{0} \frac{\partial}{\partial t}\left(\sqrt{\frac{2 G_{e} Q}{r c^{2}}\left(1+\frac{G_{e} Q}{2 r c^{2}}\right)}\right) \frac{z}{r} c \\
& +\frac{m_{0} G_{e} Q}{r^{2}}\left(1+\frac{G_{e} Q}{r c^{2}}\right) \frac{z}{r} .
\end{aligned}
$$

For a time-invariant (or very slowly changed) alpha field, the relations (45) are transformed into the form valid for the electrostatic field:

$$
\begin{aligned}
& \dot{\lambda}=0, \rightarrow F_{x}=m_{0} \ddot{x}=\frac{m_{0} G_{e} Q}{r^{2}}\left(1+\frac{G_{e} Q}{r c^{2}}\right) \frac{x}{r}, \\
& F_{y}=m_{0} \ddot{y}=\frac{m_{0} G_{e} Q}{r^{2}}\left(1+\frac{G_{e} Q}{r c^{2}}\right) \frac{y}{r}, \\
& F_{z}=m_{0} \ddot{z}=\frac{m_{0} G_{e} Q}{r^{2}}\left(1+\frac{G_{e} Q}{r c^{2}}\right) \frac{z}{r} .
\end{aligned}
$$

(f) Remarks 1 . The electrical force relations given by (45) and (46) generally describe the interactions in the strong fields. In the case of the weak fields the force relations are reduced to the well known descriptions of the interactions in the weak fields. Thus, from (46) we can see that the electrical field is a weak for $\left(G_{e} Q / r c^{2}\right) \approx 0$. In that case the term $\left(G_{e} Q / r c^{2}\right)$ can be neglected. On the other hand, the electrical field is a strong for $\left(G_{e} Q / r c^{2}\right)>>0$. For an example, in the case of the hydrogen atom the amount of this term is $\left(G_{e} Q / r c^{2}\right) \approx 5.3250 \cdot 10^{-6} \approx 0$. Thus, the electrical field of the hydrogen atom belongs to the weak fields. In the extremely strong electrical fields and extremely short distances [40-43,47], we may have situations where the $\operatorname{term}\left(G_{e} Q / r c^{2}\right)$ is close to unit $\left(G_{e} Q / r c^{2}\right) \approx 1$, or even greater than unit $\left(G_{e} Q / r c^{2}\right)>1$. For those situations the term $\left(G_{e} Q / r c^{2}\right)$ cannot be neglected.

\section{DERIVATION Of GRAVITATIONAL ForCE EQUATIONS}

Applying the substitution $M_{e g} \rightarrow M_{g}=-G M$, to the relations (43) we obtain the force equations for a particle with a rest mass $m_{0}$ valid for the gravitational field:

$$
\begin{aligned}
& \dot{\lambda} \neq 0, F_{x}=m_{0} \ddot{x}= \pm k m_{0} \frac{\partial}{\partial t}\left(\sqrt{\frac{2 G M}{r c^{2}}\left(1-\frac{G M}{2 r c^{2}}\right)}\right) \frac{x}{r} c \\
& -\frac{m_{0} G M}{r^{2}}\left(1-\frac{G M}{r c^{2}}\right) \frac{x}{r}, \\
& F_{y}=m_{0} \ddot{y}= \pm k m_{0} \frac{\partial}{\partial t}\left(\sqrt{\frac{2 G M}{r c^{2}}\left(1-\frac{G M}{2 r c^{2}}\right)}\right) \frac{y}{r} c \\
& -\frac{m_{0} G M}{r^{2}}\left(1-\frac{G M}{r c^{2}}\right) \frac{y}{r}, \\
& F_{z}=m_{0} \ddot{z}= \pm k m_{0} \frac{\partial}{\partial t}\left(\sqrt{\frac{2 G M}{r c^{2}}\left(1-\frac{G M}{2 r c^{2}}\right)}\right) \frac{z}{r} c \\
& -\frac{m_{0} G M}{r^{2}}\left(1-\frac{G M}{r c^{2}}\right) \frac{z}{r} .
\end{aligned}
$$

For the time-invariant (or very slowly changed) alpha field, the relations (47) are transformed into the form valid for the gravitational static field: 


$$
\begin{aligned}
& \dot{\lambda}=0, \rightarrow F_{x}=m_{0} \ddot{x}=-\frac{m_{0} G M}{r^{2}}\left(1-\frac{G M}{r c^{2}}\right) \frac{x}{r}, \\
& F_{y}=m_{0} \ddot{y}=-\frac{m_{0} G M}{r^{2}}\left(1-\frac{G M}{r c^{2}}\right) \frac{y}{r}, \\
& F_{z}=m_{0} \ddot{z}=-\frac{m_{0} G M}{r^{2}}\left(1-\frac{G M}{r c^{2}}\right) \frac{z}{r} .
\end{aligned}
$$

\section{B. Proof of the Prediction c) of RAF Theory}

The gravitational force relations, given by (47) and (48), generally describe the interactions in the strong fields. In the case of the weak fields the force relations (47) and (48) are reduced to the well known descriptions of the interactions in the weak fields. Thus, from the relations (48) we can see that the gravitational force is negative (attractive) for $\left(G M / r c^{2}\right)<1$. This is the case in the weak gravitational field, where the term $\left(G M / r c^{2}\right)$ can be neglected. On the other hand, the gravitational force becomes positive (repulsive) if $\left(G M / r c^{2}\right)>1$. This is the case in the extremely strong gravitational field [40-43,47], where the term $\left(G M / r c^{2}\right)$ cannot be neglected. In our solar system the term $\left(G M / r c^{2}\right)$ is too small and can be neglected. For an example, on the surface of our Sun the amount of this term is $\left(G M / r c^{2}\right) \approx 2.1193 \cdot 10^{-6}<<1$. On the surface of our planet Earth the related influence of the Sun to this term is $\left(G M / r c^{2}\right) \approx 0.989 \cdot 10^{-8}<<1$. Including mass and radius of the planet Earth in this term we obtain that the related gravitational influence of the planet Earth on its surface is $\left(G M / r c^{2}\right) \approx 0.695 \cdot 10^{-9}<<1$. The presented amounts of the term $\left(G M / r c^{2}\right)$ in our solar system could be the answer to the question: why our experience is that gravitational force is only negative (attractive) force?

Further, if the term $\left(G M / r c^{2}\right)=1$, then the gravitational force is equal to zero. This is happened at the gravitational radius $r=\left(G M / c^{2}\right)$. This radius separates the attractive and repulsive forces in a gravitational field. At the minimal radius $r_{\min }$ gravitational force is positive (repulsive) $F_{r_{\text {min }}}=\left(4 m_{0} c^{4} / G M\right)$. We can say that the nature protects itself from the singularity by producing positive (repulsive) gravitational force $F_{r_{\text {min }}}$ at minimal radius $r_{\min }$. From the relations (48) we can see that the gravitational force is positive (repulsive) in the region $r_{\text {min }} \leq r<r_{0}$, where $r_{\text {min }}=\left(G M / 2 c^{2}\right)($ see the second part of this theory [31] $)$ and $r_{0}=\left(G M / c^{2}\right)$. At the Schwarzschild radius $r_{s c h}=\left(2 G M / c^{2}\right)$ the gravitational force is negative (attractive) $F_{r_{s c h}}=-\left(m_{0} c^{4} / 8 G M\right)$ and belongs to the negative (attractive) set of gravitation forces in the region $r_{0}<r \leq \infty$.

On the Planck's scale one can substitute $M$ by Planck's mass $M_{p}$ and $r$ by Planck's length $L_{p}$. Thus, on the Planck's scale the term $\left(G M_{p} / L_{p} c^{2}\right) \approx 1$. Following the relations (48) we can conclude that the gravitational force of the Planck's mass $M_{p}$ is positive (repulsive) if the radius $r$ is smaller than the Planck's length $L_{p}, r<L_{p}$. On the other hand, if the radius $r$ is greater than the Planck's length $L_{p}$, $r>L_{p}$, then the gravitational force of the Planck's mass $M_{p}$ is negative (attractive).

The previous consideration theoretically confirms the prediction c) of the RAF theory: the gravitational force becomes positive (repulsive) if $\left(G M / r c^{2}\right)>1$ that could be a source of a dark energy. Of course, this should be proved by the related experiments.

\section{CONCLUSION}

It is well known that General Relativity Theory (GRT) cannot be applied to the extremely strong gravitational field at the Planck's scale, because of the related singularity. Here we show that Relativistic Alpha Field (RAF) theory extends the application of GRT to the extremely strong fields at the Planck's scale. Further in this paper we considered and theoretically proved two of the four predictions of RAF theory: c) the gravitational force becomes positive (repulsive) if $\left(G M / r c^{2}\right)>1$ that could be a source of a dark energy, and d) unification of electrical and gravitational forces can be done in the standard four dimensions (4D). It is also shown that positive (repulsive) gravitational forces belong to the extremely strong gravitational fields. On the other hand, negative (attractive) forces belong to the weak gravitational fields, like in our solar system. This could be the answer to the question: why our experience is that gravitational force is only negative (attractive) force? Further, it is presented that at the minimal radius $r_{\min }$ gravitational force is positive (repulsive) $F_{r_{\text {min }}}=\left(4 m_{0} c^{4} / G M\right)$. Therefore, we can say that the nature protects itself from the singularity by producing positive (repulsive) gravitational force $F_{r_{\text {min }}}$ at minimal radius $r_{\text {min }}$. If RAF theory is correct, then it could be applied to the both weak and strong fields at the Universe and Planck's scales giving the new light to the regions like black holes, quantum theory, high energy physics, Big Bang theory and cosmology. Of course, the predictions of RAF theory should be proved by the related experiments.

\section{ACKNOWLEDGMENTS}

The author wishes to thank to the anonymous reviewers for a variety of helpful comments and suggestions. This work is supported by grants (120-1201842-3048) from the National Scientific Foundation of Republic of Croatia.

\section{REFERENCES}

[1] A. Einstein, Ann. Phys. 49, 769-822 (1916).

[2] A. Einstein, The Meaning of Relativity (Princeton Univ. Press, Princeton, 1955).

[3] C. Sean, Spacetime and Geometry: An introduction to General Relativiry (Amazon.com Books.htm, Hardcover, 2003). 
[4] S. Weinberg, Gravitation and Cosmology: Principles and Application of the General Theory of Relativity (Gebundene Ausgabe, RelEspWeinberg.pdf., 1972).

[5] S. W. Hawking, G. F. R. Ellis, The Large Scale Structure of Space-Time (Univ. Press, Cambridge, 1973).

[6] M. Blau, Lecture Notes on General Relativity (A. Einstein Center for Fundamental Physics, Univ. Bern, Bern, 2012, 2014).

[7] T. Kaluza, Zum Unitätsproblem in der Physik (Sitzungsber. Preuss. Akad. Wiss., Berlin, 1921).

[8] O. Klein, Z. Phys. A 37, 895-906 (1926).

[9] E. Witten, Nucl. Phys. B 186, 412-428 (1981).

[10] T. Appelquist, A. Chodos, and P. G. O. Freund, Modern Kaluza-Klein Theories (Addison-Wesley, Menlo Park, Cal., 1987).

[11] M. J. Duff, Kaluza-Klein Theory in Perspective (Proc. of the Symposium: The Oskar Klein Centenary, World Scientific, Singapore, 22-35, 1994).

[12] J. M. Overduin, and P. S. Wesson, Phys. Rep. 283, 303-378 (1997).

[13] P. S. Wesson, Space-Time-Matter, Modern Kaluza - Klein Theory (World Scientific, Singapore, 1999).

[14] P. S.Wesson, Five-Dimensional Physics: Classical and Quantum Consequences of Kaluza-Klein Cosmology (World Scientific, Singapore, 2006).

[15] D. Z. Freedman, and A. Van Proeyen, Supergravity (Cambridge Univ. Press, Cambridge, 2012)

[16] J. Wess, B. and A. Zumino, Phys. Lett. B 49, 52 (1974).

[17] M. K. Gaillard, and B. Zumino, Nucl. Phys. B 193, 221 (1981).

[18] M. B. Green, J. H. Schwarz, and E. Witten, Superstring Theory (Cambridge Univ. Press, Cambridge, 1987).

[19] J. Polchinski, String Theory (Cambridge Univ. Press, Cambridge, 1998).

[20] R. Brandenberger, and C. Vafa, Nucl. Phys. B 316, 391-410 (1989).

[21] N. Arkani-Hamed, A. G. Cohen, and H. Georgi, Phys. Rev. Lett. 86, 4757 (2001).

[22] C. T. Hill, S. Pokorski, and J. Wang, Phys. Rev. D 64, 105005 (2001).

[23] C. Cshaki, G. D. Kribs, and J. Terning, Phys. Rev. D 65, 015004 (2002).

[24] E. C. Poggio, H. R. Quinn, and S. Weinberg, Phys. Rev. D 13, 1958 (1976).

[25] T. R. Taylor, and G. Veneziano, Phys. Lett. B 212, 147 (1988).

[26] H. C. Cheng, B. A. Dobrescu, and C. T. Hill, Nucl. Phys. B 573, 597 (2000).

[27] C. Cshaki, J. Erlich, C. Grojean, and G. D. Kribs, Phys. Rev. D 65, 015003 (2002).

[28] N. Arkani-Hamed, and M. Schmaltz, Phys. Rev. D 61, 033005 (2000).

[29] M. Gogberashvili, Europhys. Lett. 49, 396 (2000).

[30] B. M. Novakovic, Relativistic alpha field theory - Part I. To be published in IJNTR (2015).

[31] B. M. Novakovic, Relativistic alpha field theory-Part II. To be published in IJNTR (2015).

[32] B. M. Novakovic, Int. J. of Comput. Anticip. Syst., IJCAS 27, p. 93 (2014).

[33] S. Gallot, D. Hullin, and D. J. Lafontane, Riemannian Geometry ( Springer-Verlag, Berlin, New York, ed. 3, 2004).

[34] C. T. J. Dodson, and T. Poston, Tensor Geometry. Graduate Texts in Mathematics (Springer-Verlag, Berlin, New York, ed. 2, 1991), p. 130

[35] M. T. Vaughin, Introduction to Mathematical Physics (Wiley-VCH Verlag GmbH \& Co., Weinheim, 2007).

[36] B. M. Novakovic, in Proceedings of the Ninth Int. Conf. on Comp. Anticip. Syst., Liege, 2009, edited by D. Dubois (University of Liege, Liege 2009), AIP-CP 1303, p. 141 (2010). DOI: 10.1063/1.3527149.

[37] P. A. M. Dirac, Directions in Physics (Wiley, New York, 1978).

[38] I. Supek, Theoretical Physics and Structure of Matter, Part I (Skolska knjiga, Zagreb, 1992).

[39] I. Supek, Theoretical Physics and Structure of Matter, Part II (Skolska knjiga, Zagreb, 1990).

[40] D. H. Perkins, Introduction to High Energy Physics (Cambridge Univ. Press, Cambridge, 2000).

[41] D. Sherman, et al., Nat. Phys. 11, 1-5 (2015).

[42] J. Steinhauer, Nat. Phys. 10, 864-869 (2014).

[43] M. Meckel, et al. Nat. Phys. 10, 594 - 600 (2014)

[44] B. M. Novakovic, D. Novakovic, and A. Novakovic. in Proceedings of the Sixth Int. Conf. on Comp. Anticip. Syst., Liege, 2003, edited by D. Dubois (University of Liege, Liege 2003), AIP-CP 718, p.133 (2004). DOI: $10.1063 / 1.1787318$

[45] B. M. Novakovic, D. Novakovic, and A. Novakovic, in Proceedings of the Seventh Int. Conf. on Comp. Anticip. Syst., Liege,
2005, edited by D. Dubois (University of Liege, Liege 2005), AIP-CP 839, p.144, (2006). DOI: 10.1063/1.2216624.

[46] B. M. Novakovic, Strojarstvo 53 (2), 103-111 (2011).

[47] R. Ding, et al. Phys. Rev. D 92, (015008) (2015).

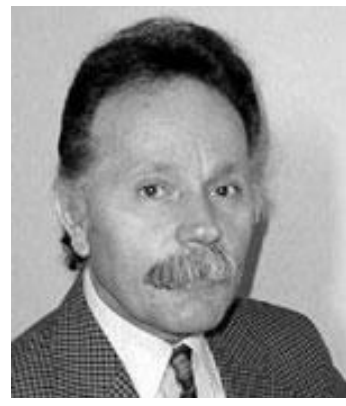

Branko Novakovic is a Professor emeritus at FSB - University of Zagreb, Croatia. Prof. Novakovic received his $\mathrm{PhD}$ from the FSB-University of Zagreb in 1978. His research interests include physics, control systems, robotics, neural networks, and fuzzy control. He is author of two books, Control Systems (1985) and Control Methods in Robotics, Flexible Manufacturing Systems and Processes (1990), and the first co-author of a book Artificial Neural Networks (1998). He has published over 220 research papers in his research of interest. 